\title{
(3) \\ riscos \\ IntErgy: Minimization of Interference and Energy Optimization in Multi-hop Wireless Networks
}

\author{
Kulangara Silpa Prabhu, A. Felix Arokya Jose \& V. Jeya Krishnan \\ Dept. of Computer Science and Engineering, Karunya University, Coimbatore, India
}

\begin{abstract}
Wireless networks are made up of several nodes or cluster of nodes which are randomly or arbitrarily distributed in an environment. These cluster of nodes interact with each other inorder to exchange data. The concept of cooperative communication in a Virtual Multiple Input Single Output environment is used here for this purpose. Since signals travel a long distance it can undergo fading due to interference. As a result the quality of the signal reduces at the other side. A cluster diversity protocol merely captures the transmission at the source part which also optimizes throughput and improves performance thereby minimizing interference.
\end{abstract}

Keywords-cluster nodes, cooperative communication, interference, multi-hop networks, signal fading.

\section{INTRODUCTION}

In networking, the nodes in a cluster communicate with each other in a co-operative and non-cooperative way. Here we focus on cooperative communication in networks. Generally, more than one antenna is required at source part to transmit. Thus a single antenna can be used in a multi-user environment. This antenna is shared. Thus a virtual multiple antenna is generated at transmitter side. Single antenna mobiles take advantage of this communication. Due to sharing Virtual Multiple Input Single Output (VMISO) is obtained. Each node cooperate with each other to improve QoS. VMISO can broadcast easily to cooperating nodes with a single transmission. It improves transmission and thus improves communication performance.

Bringing cooperation among the network nodes and giving tolerance to interference has been a challenge throughout this area. Cooperation also aims at attaining cooperation gain which includes bringing up power gain and diversity gain.

The next section focuses on problems in two areas, that is, single in and single output (SISO) and also the VMISO without cooperative communication[1].
The following section gives the description about the Cluster diversity algorithm and also about how the cluster diversity protocol gives us Optimized way of Minimizing Interference (OpMInter). We also present the benefits and drawbacks of few existing systems[7].

\section{PROBLEM STATEMENT}

Single Input Single Output (SISO) consists of a single antenna at the source and a single antenna at the receiver. Transmission through SISO path is clearly disadvantageous [1]. This is mainly because (in networking area) when a single transmitter node transmits signal to a single receiver node, the signal has to travel a long distance. Thus it gets affected by interference and thus the end-to-end throughput decreases. The main reason is that since there is only a single path, we cannot guarantee that the signal can reach the other side as in Fig.1

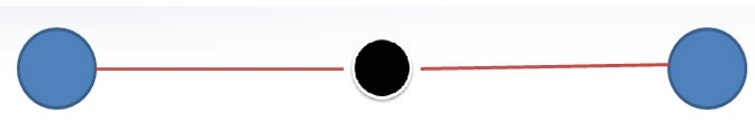

Fig. 1 Single Input Single Output

Virtual Multiple Input Single Output (VMISO) consists of a cluster of nodes at the transmitter side. It has got better performance benefits than SISO and also since each node distributes flows there is guarantee that any one signal can reach the receiver part. Thus, the rate increases but total time taken for each hop is now increased. Clusters are also found out to be fixed in size and also the lack of cooperation among nodes further sustains the interference problem in VMISO which is again a drawback.

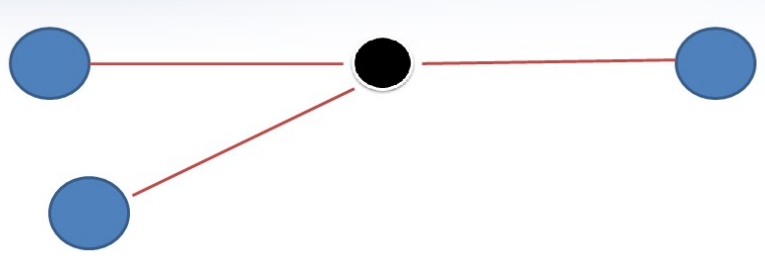

Fig.2 VMISO without cooperation 
Thus we try to bring cooperation among the cluster of nodes along with the benefits of VMISO. Thus VMISO with cooperative transmission is introduced here, which will further minimize the interference in the arbitrary topology.

\section{RELATED WORKS}

A distributed cooperative forwarding framework based on randomized coding is proposed in [1], where cooperative links are formed and packets are forwarded from one place to another, without explicit relay selection, actuation or resource allocation[1]. Here a Cooperative Forwarding within Progress Region (CFPR) scheme is used. In this scheme, the packets are progressively forwarded from source to destination, by cooperative transmissions of the nodes, which reside in a predefined region[6]. Its advantages are: (i) packet is delivered to target correctly. (ii) reduction in total number of hops. (iii) guaranteed transmission in case of mobility or fading. But disadvantage is that packet delivery ratio decreases with time.

In multi-hop ad hoc networks, stations may pump more traffic into the networks than can be supported, resulting in very high packet-loss rate, instability in re-routing and unfairness problems[2]. This paper shows that controlling the offered load at the sources can eliminate these problems.

An analysis on throughput is made here based on hidden node problems. Its advantages are: (i) retransmission of a node more than once is reduced. (ii) sustainable throughput increases as hidden node problem decreases. (iii) link failure chance is decreased. (iv) packet loss ratio is less. And drawbacks include (i) complicate analysis and chance of collision increases. (ii) as number of node increases, optimal offered load decreases.

[3] found optimal cooperator selection policies for multihop networks with MIMO transmissions. The cooperator selection process was modeled for arbitrary topologies through a suitable Markov chain[3]. Hence, this chain was reduced according to an original pruning technique which cuts states with negligible impact on the optimal solution[3]. Its advantages are : (i)optimal cooperator selection for links affected by path loss.(i)optimal cooperator selection for links affected by multipath fading. But delay in small amount occurs due to cooperator selection policy and also SNR is kept minimum due to optimal selection of links.

A new high performance forwarding scheme is used in [4] that is Transmit Diversity based Cooperative Opportunistic Routing (TDiCOR), that efficiently exploits multi-user and transmit diversity to improve the overall throughput in wireless multi-hop networks [4]. Its benefits are: (i)error rate is minimized.(ii)reliability in acknowledgment.(iii)SNR in small amount $10 \mathrm{db}$. But disadvantage is that the gain and loss of transmit diversity varies with bit rate.

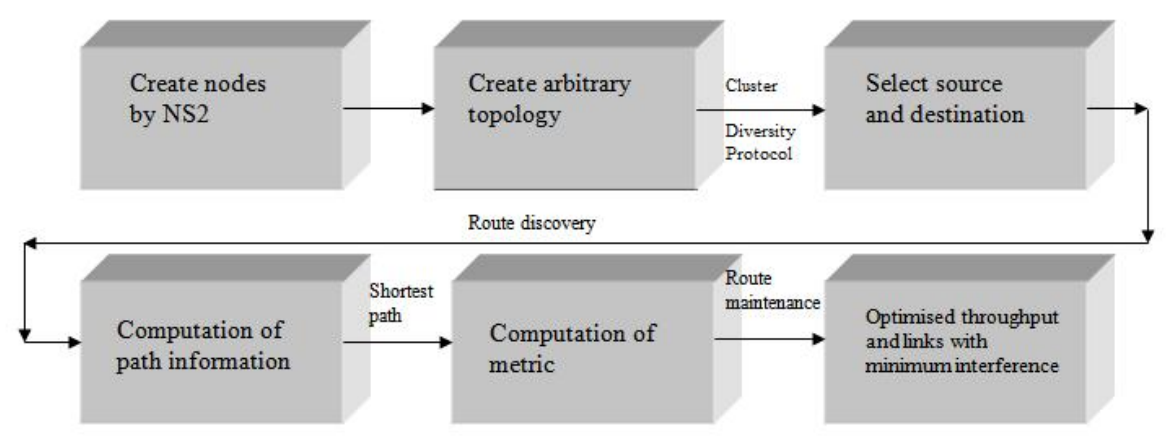

Fig.3 OpMInter Architecture

A set of iterative steps are involved in Feasible Solution Algorithm[5]. During the first iterative step, an upper bound on the objective value is obtained by solving a relaxed version of the MILP problem. The solution may become fractional. Therefore, a local search algorithm, called Feasible Solution Construction (FSC), is proposed to obtain a feasible solution from the relaxed solution. The feasible solution obtained from FSC provides a lower bound on the objective value. FSC algorithm is an efficient polynomial time algorithm, and addresses the solution construction process in three phases, namely, Path Determination, CR Assignment, and Flow Recalculation[5]. Its advantages are: (i) increases capacity of wireless networks. (ii)solve joint optimization problem of relay nodes and flow routing. (iii)rate gain. (iv)antenna systems with $\mathrm{CC}$ do not have to equip multiple antennas on each node in network and so each node has only a single antenna. (v)spatial diversity is achieved. (vi)higher throughput obtained. Drawbacks include: (i)usage of orthogonal channel because only few users are transferring data simultaneously. (ii)delay and time consuming in performing these operations.

\section{CLUSTER DIVERSITY ALGORITHM}

An arbitrary topology is taken in this case. Consider cluster of nodes at sender side and single node at the receiver side and an intermediate nodes as in Fig 4. 
Since we concentrate more on source side, we consider the cluster of nodes and first intermediate node as the MISO environment. Steps involved in this algorithm are (i)Compute Path Information and (ii) Compute Metric.

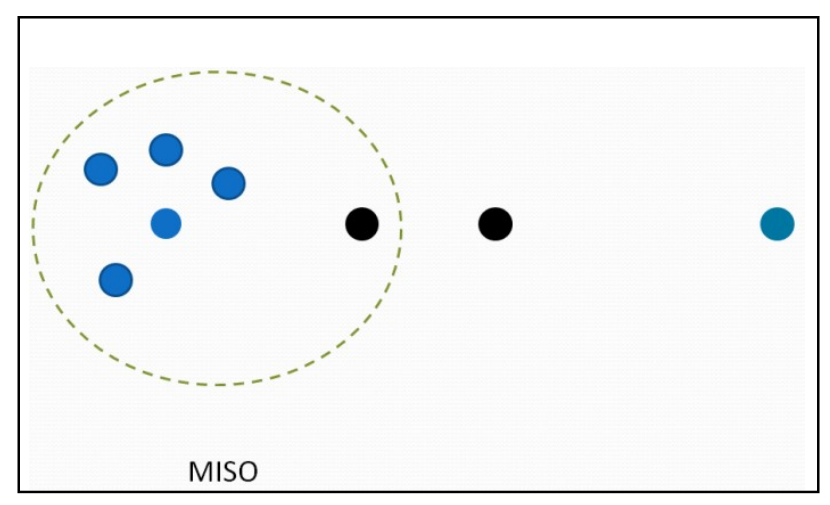

Fig 4. Initial Topology with VMISO alone

Since VMISO alone is not advantageous as explained earlier, an integration of VMISO along with cooperative communication is considered in the same topology. For this purpose we choose an initialization node (green node as in Fig 5), from among the cluster of nodes at the source side as shown in Fig 5

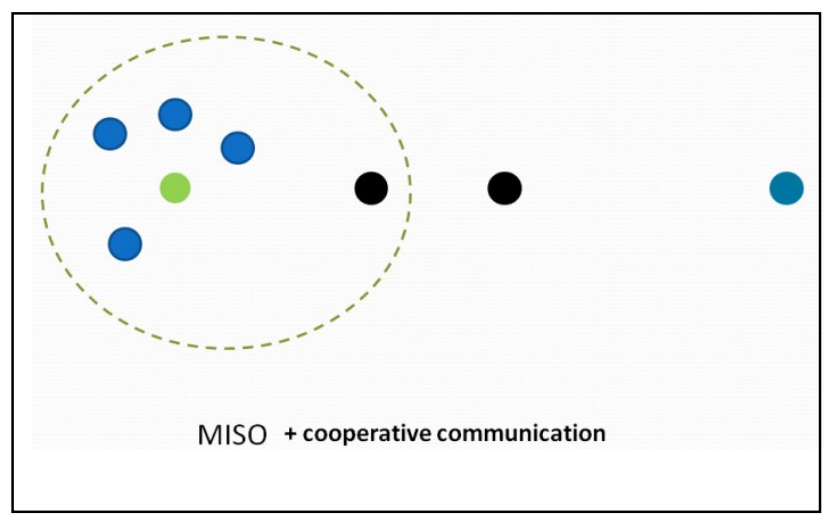

Fig 5. Initial Topology with cooperative communication added

\subsection{Compute Path Information.}

Here the inputs given are source and destination. By using any shortest path method find out the shortest path. It is better to use the best and efficient method of shortest path algorithm.

\subsection{Compute Metric}

Give the shortest path and modulation order as input in this case. Inorder to compute the metric we need to verify whether end-to-end path is available or not. If it is not available, we need to go for the next shortest path.

Otherwise, compute the interference measure. Now, we need to check whether this interference affects the data flow or not and accordingly the cluster size has to be changed. Finally, and optimized link with optimized throughput is obtained as the result.
These steps have been shown in the architecture Fig3. This clearly shows the end result, that is minimized interference.

\section{MINIMIZING INTERFERENCE}

There are several internal and external methods that affect interference. The internal sources in our topology are the total number of relay nodes or nodes in cluster $N_{r}$, Traffic per each node $N_{T}$, Hop count $H_{c}$ and external sources in our natural world.

$$
\text { Internal Sources }=N_{r}+N_{T}+H_{c}+\text { External Sources. }
$$

After bringing cooperation among the relay nodes or cluster of nodes and by applying cooperation strategy (which focuses on power gain $P_{G}$ ), the interference will be almost negligible since then signal reaches with larger strength at the receiver side. Thus optimized throughput is obtained along with minimized interference.

\section{ARCHITECTURE}

As already mentioned, an arbitrary topology is considered in this case. We assume cluster of nodes at sender side and single node at the receiver side. There are two major processes involved (i)Route discovery and (ii)Route maintenance Fig 3.

\subsection{Route Discovery}

This process checks whether end-to-end path is available or not. This is done by sending and receiving certain commands like route request and route reply.

\section{A. Route Request (RREQ)}

RREQ is the first step in route discovery phase. Here the cluster of nodes send the request to neighbouring nodes and wait for reply in order to check if the neighbouring nodes are active or ready to send at that particular moment. This also tries to maintain cooperation among the cluster of nodes.

In RREQ packet the metrics are received signal strength, interference level, neighbour list number of flows already served. The interference information is obtained by nodes monitoring which channel is busy.

\section{B. Route Response (RREP)}

This packet is a response to the RREQ. In RREP the packet contains source id and path. After the computation of metric the output obtained is optimized path with less cluster size and modulation order.

\subsection{Route Maintenance}

Finally route maintenance is done as shown in Fig.3. When a route is failed, new route is recomputed without much delay. The overhead is also kept to a minimum with the help of a MAC protocol.

\section{RESULTS}

In the whole process, the cooperation is maintained among the cluster of nodes with the help of a pilot tone 
[1] being send from initial node to its neighbours in its cluster. This keeps all the neighbours active or checks whether the nodes are active or not when the initial node gets a response back as shown in Fig 6.
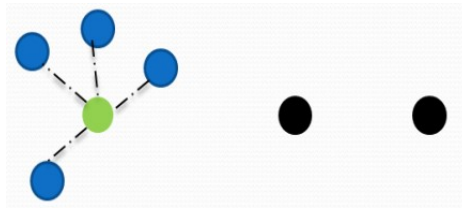

Fig 6. Sending and Receiving of Pilot Tones

After the cooperation among the cluster of nodes are confirmed the major advantage is the increase in performance and throughput. Next, the nodes including the initialization node sends the signal to the first intermediate node after checking the path availability Fig 7.The process of sending signal continues to all other nodes till the receiver side is reached where we find the added advantage of increase in performance. This also includes a new strategy called cooperation strategy [1] which includes increase in the power of signal which is also major factor of performance gradation.

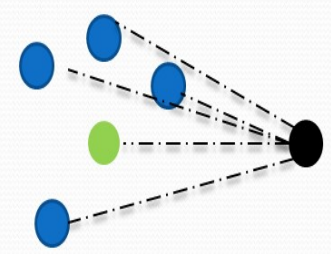

Fig. 7 Cooperation Strategy

As we can see that all the existing systems that use SISO and VMISO without cooperation is pulled down in terms of its performance at the end by the effect of interference. As a result the throughput also gets affected. We also observe the amount of energy consumed by nodes when the signal is transmitted, which is found out to be high in terms of Joules[7]. The interference can occur as a result of internal and external sources as explained earlier and as illustrated in Fig.8.

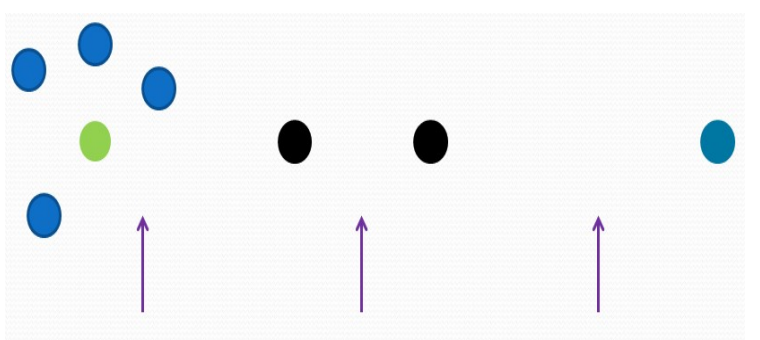

Fig. 8 Interference prone area taken care of

To overcome the above mentioned problems, the optimized method of using a cluster diversity protocol is used. Thus the nodes consume only less energy as shown in Fig 9. This is due to the involvement of cooperation between the cluster of nodes at the transmitter side. Thus the interference is minimized comparatively

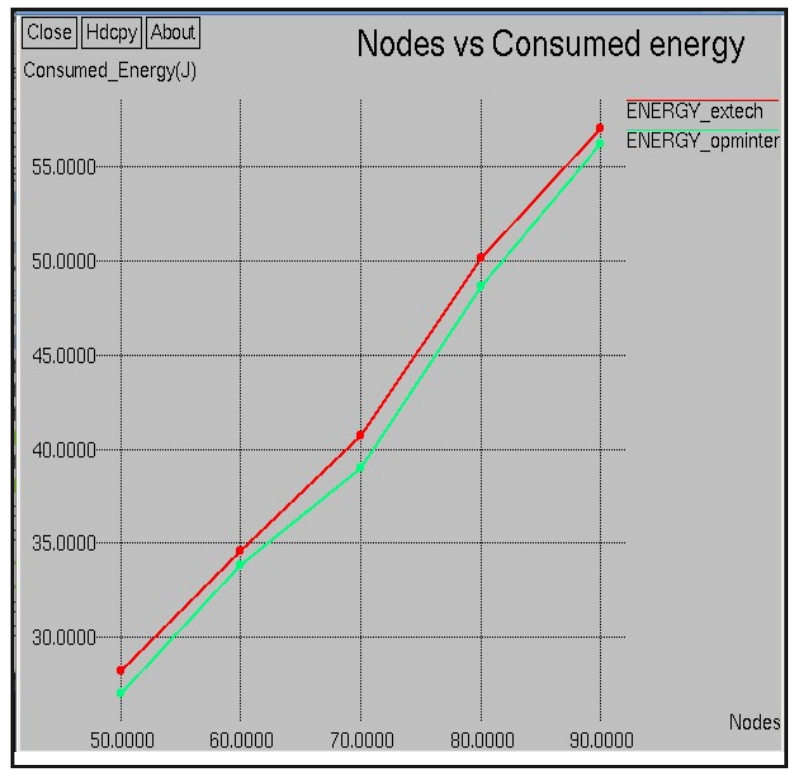

Fig.9 Existing technology Energy Vs OpMInter Energy

The graph Fig.9 shows the amount of energyconsumed by different number of nodes.It can be clearly seen that the existing technology consumes much more energy than the proposed OpMInter method. Say for example, 50 nodes consume only $23.4 \mathrm{~J}$ of energy in our method (green line) whereas in existing method (red line) the energy consumption is $26.7 \mathrm{~J}$. Likewise the graph depicts the energy consumption among 60 to 90 nodes comparing both technologies as explained above

\section{CONCLUSION}

The conclusion of this work is to improvise the cooperative transmission in wireless networks, in terms of better scalability and performance. We tried to improvise the performance by focusing on the transmitter side, and by inducing cooperation between the cluster of nodes in a VMISO environment we also reduced interference. Cooperation strategy plays an important role. This also benefited the efficient usage of energy and optimization of throughput. The interference prone areas were taken much care inorder to benefit increase in throughput and data rate.

Further enhancement can be focused on the source side and the optimization of nodes inorder to increase the data rate, packet delivery ratio etc.

\section{REFERENCES}

[1] Kulangara Silpa Prabhu, A.Felix Arokya Jose, "Clad: Accurate Capture Of Transmission In multi-hop Wireless Networks," in IJERT, January 2013, Vol 2, Issue 1.

[2] P. C. Ng and S. C. Liew, "Throughput analysis of ieee802.11 multi-hop ad hoc networks," IEEE/ACM Transactions on Networking (TON), pp. 309-322, 2007.

[3] M. Rossi, C. Tapparello, and S. Tomasin, "On optimal cooperator selection policies for multi- 
hop ad hoc networks," IEEE Transactions on Wireless Communications , pp. 506-518, 2011.

[4] M. Kurth and et al, "Cooperative opportunistic routing using transmit diversity in wireless mesh networks," in IEEE INFOCOM, 2008.

[5] S. Sharma, Y. Shi, T. Hou, H. D. Sherali, and S. Kompella, "Cooperative communications in multi-hop wireless networks: Joint flow routing and relay node assignment," in IEEE INFOCOM, 2010, pp. 1-9.
[6] Y. Xiao, Y. Guan, W. Chen, C.-C. Shen, and L. Cimini, "Location-aware cooperative routing in multihop wireless networks," in IEEE WCNC, 2011, pp. 761-766.

[7] Kulangara Silpa Prabhu, A.Felix Arokya Jose, V.Jeya Krishnan, "OpMInter: An Optimization Method to Minimize Interference in Multi-hop Wireless Networks," International Conf by IRD India, January 2013, ISBN: 978-93-81693-88-6. 